\title{
Functional effects of food: what do we know in children?
}

\author{
Peter J. Aggett \\ Lancashire School of Health and Postgraduate Medicine, University of Central Lancashire, Preston PRI 2HE, UK
}

\begin{abstract}
The knowledge base underpinning the setting of nutrient requirements for children and adolescents is not very secure. The advent of the concepts of optimal nutrition and functional foods has encouraged the possible use of functional effects as criteria for adequate nutrition in this age group. Target functions have been identified for growth development and differentiation and for behavioural and cognitive development. However, ideal markers or effects for these are not generally available. Additionally, nutrition in young people should avoid predisposing them to diet-related disease in later life. It is suggested that functional effects should include markers of reduction of risks of disease (nutritional safety) as well of benefits for health and well-being. Such markers of functional effects should be expected to arise from fundamental studies of nutrient-gene interactions and post-genomic metabolism.
\end{abstract}

Children: Food safety: Functional food: Nutrition

It is appreciated that marginal nutrient supplies are more critical during development and maturation than when, ideally, an individual's full potential has been achieved, and this has been emphasised in an earlier assessment of functional outcomes and food effects in childhood and adolescence (Koletzko et al. 1998). It is particularly important during this period to be concerned about delivering not only single nutrients but also the appropriate mix of nutrients at critical periods when they are needed. Furthermore, in considering the supply of nutrients and other food components to maturing individuals, attention needs to be paid not only to immediate and short-term effects but also to the longer-term impact of early nutrition, in the context of both optimal development and minimising or avoiding risks of diet-induced metabolic dysfunction and disease. Thus attention needs to be paid to the borderline between physiological adequacy and excess that might not have any immediate effects arousing toxicological concern, but which might nevertheless have a long-term adverse impact.

The phenomenon of 'metabolic programming' has caught the imagination in understanding the effects of nutrient exposure in early life, including while in utero, on the risk of developing metabolic syndrome, coronary heart disease, hypertension and hypercholesterolaemia in adult life (Barker, 1994; Godfrey \& Barker, 2001). In fact evidence, some of which is based on prospective studies, is emerging of these disorders manifesting before adulthood.

An example of this is the excess caloric intake relative to energy expenditure and the increasing prevalence of excessive weight gain, overweight, insulin resistance, obesity and metabolic syndrome in younger people (Deckelbaum \& Williams, 2001). Another possible example is the positive correlation of $\mathrm{Na}$ intake with blood pressure in the developmental years (Geleijnse et al. 1990, 1996).

One can suppose that, for such problems to develop, intakes of the nutrients and food components concerned would have to exceed the capacity of the relevant customary systemic homeostatic and adaptive mechanisms. Thus in considering functional outcomes as indicators of appropriate nutritional intakes, particularly, but not exclusively, in children and adolescents, one needs to consider intakes within a range appropriate to homeostatic maturation and capacity.

Food is a cocktail of natural and man-made components, each with a specific dose-related spectrum of potential physiological, pharmacological, adverse and toxicological effects. Currently there is particular interest in the possibility that components other than traditional nutrients might have beneficial effects, and that even for traditional or accepted nutrients, inappropriate end-points have been used to estimate recommended intakes; as a consequence important functional benefits which might arise from higher intakes have been overlooked.

The outcomes used in assessment of requirements have, of necessity, varied according to the current knowledge of the functions and metabolism of the nutrient being considered. For most nutrients there are few appropriate dose-response studies to facilitate the identification of threshold levels and events that could be used to determine safe ranges of intake and to derive dietary reference values. The criteria used to assess reference values, and the values set, have been seen to relate to avoiding deficiency, and to use a pragmatic blend of functional, homeostatic and indicators of body burden to derive reference intakes. Occasionally the dearth of evidence has meant that customary or 
observed intake at which health appears to be maintained has had to be used (Committee for Medical Aspects of Food Policy, 1991).

It has long been accepted that the science base for deriving reference values is inadequate, and part of the reaction to this has been the proposal that functional outcomes, or more general manifestations of good health, should provide the basis for discerning an adequate diet and nutrient intake. On this basis, observed intakes or intakes in excess of these reference values are advocated to achieve optimal nutrition: one wonders if this could be dubbed a 'precautionary approach'. Even so, these intakes need to be responsible and evidence-based. Concern in this regard underlies the recent exercises in assessing 'safe upper levels' in the UK (Expert Group on Vitamins and Minerals, 2003) or 'tolerable upper intake levels' by the Scientific Committee on Food in the EU for intakes for vitamins and minerals; here again, however, the evidence basis on which to base such levels is not strong.

For the cynic, the spectrum of functional outcomes and requirements is relative, and can be envisaged to extend from survival or reducing the risk of death from undernutrition to the absence of adverse effects due to any specific deficiency ('health maintenance'), and to the promotion of better health and well-being and the reduction of the risk of disease. The latter end of this spectrum is where functional foods are thought to have potential roles.

A food can be regarded as functional if it can be demonstrated to affect beneficially one or more target functions in the body, beyond adequate nutritional effects, in a way that is relevant to an improved state of health and well-being, the reduction of risk of disease or both; or if the health claim can be made for the specific effects of the food or its component(s) (Diplock et al. 1999). The challenge with this definition is understanding what 'adequate nutritional effects' are, and how they might be objectively determined, particularly since good-quality information about both adequate nutritional effects and functional nutritional effects would ultimately be derived from a sound evidence-based approach to nutrition, drawing on traditional strategies characterising dose-response relationships and the identification of markers that would enable the adequacy or otherwise of intakes to be judged. This ethos underpinned a European approach to exploring functional effects and functionality of foods.

The background concepts of functional food effects are that diet and food components modulate various functions in the body, and that they participate in the maintenance of good health to the extent that they promote well-being and health, and reduce the risk of some diseases. The identification of possible functional effects derives from the exploration of hypotheses generated by epidemiological or ecological studies, which have demonstrated possible links between diets and dietary components and health effects. The need for an evaluative or hypothesis-led approach to these associations was the basis of the Functional Food Science Concerted Action (FUFOSE; Diplock et al. 1999), particularly since further experimental analysis of epidemiological associations did not always support assumptions derived from epidemiological observations about, e.g., the benefits of $\beta$-carotene or dietary 'fibre' (Gerber, 2001).

Table 1. Examples of opportunities for modulation of target functions related to growth, development and differentiation by candidate food components with possible markers (Diplock et al. 1999)

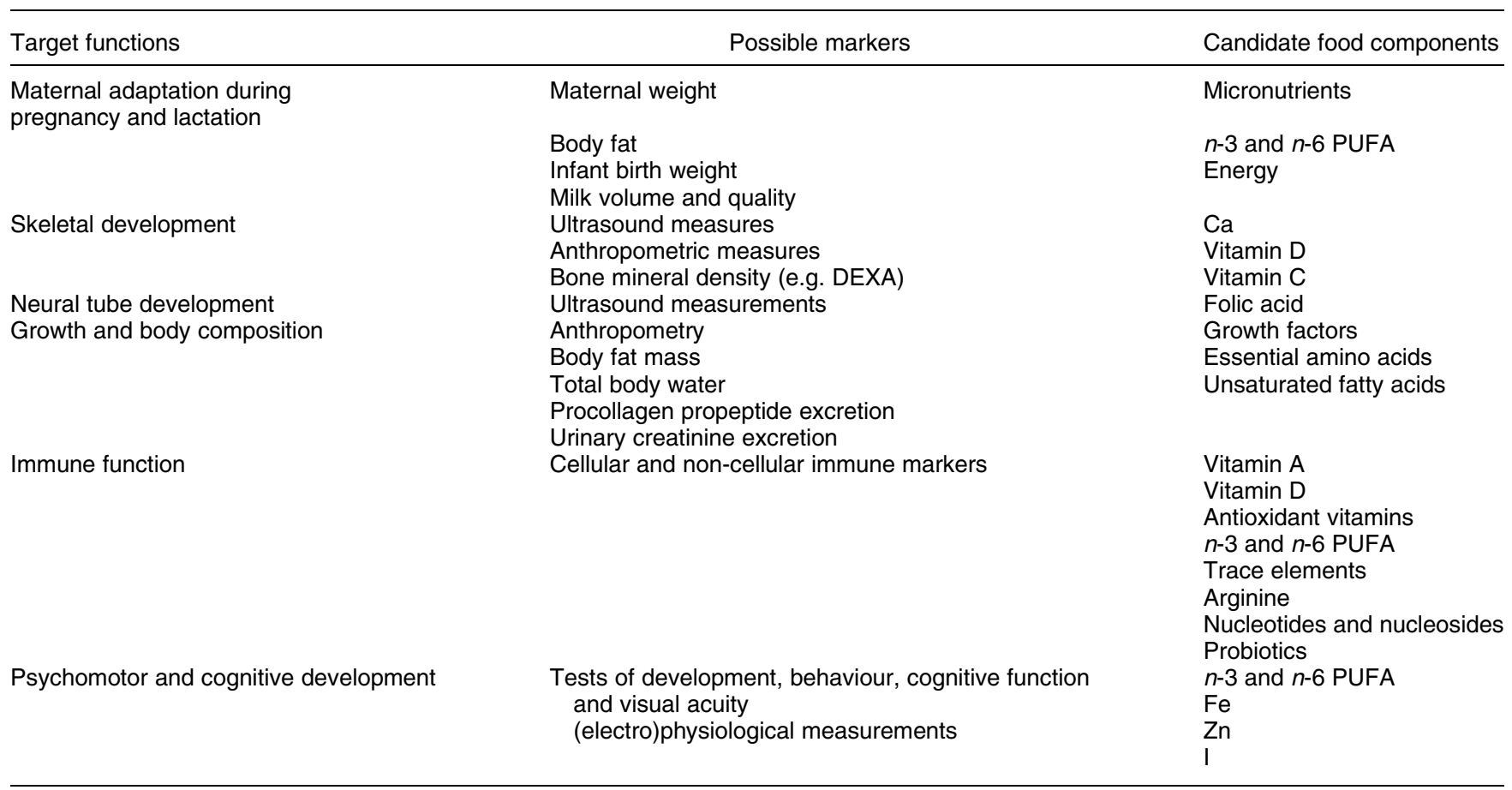


The purposes of FUFOSE were:

1. To establish a science-based approach for concepts and functional food science.

2. To examine the available science from a functiondriven point of view rather than a component- or product-driven perspective.

3. To assess critically the science base required to provide evidence that specific nutrients, or food components, positively affect functions.

There is therefore some congruence between functional food science and the use of functional effects, or efforts to identify functional effects, to determine reference nutrient intakes in children. Some target functions and related markers identified by the FUFOSE subgroup for 'Growth, development and differentiation: a functional food science approach' for children through to adolescence are summarised in Table 1. It would also be valid to include target functions related to behaviour and cognition as well (Bellisle, 2004). The target functions belong to key categories of development, but are broad, and the markers vary in their specificity and selectivity, their quality assurance characteristics and in the availability of universally applicable reference values. Some of these problems arise from the multiplicity of nutrients and food components that can affect the markers and target functions, and also the vulnerability of the outcomes to other confounders.

The functional effects shown are oriented towards health and well-being rather than the reduction of disease. None the less, as has already been noted, the pathogenesis of diet-related adult disease and metabolic dysfunction can start in early life. On this basis it would seem important to be interested in the effect of childhood nutrient intakes on target functions and markers relevant to the reduction of risks of disease. These would include outcomes relevant to substrate metabolism, dysfunction and damage arising from reactive oxidative species, and cardiovascular function (Diplock et al. 1999), and it would seem legitimate to use markers related to these target functions to monitor the safety of any dietary interventions in young people. However, because of the latency of some adverse effects, it might be necessary to use early biochemical and metabolic functional markers, for example of increasing insulin resistance, rather than markers such as weight and BMI. In children, trends or temporal changes in markers might be as important as getting values outwith reference ranges.

The additional dimension in children is identifying the critical periods at which their target functions are susceptible to nutritional or dietary interventions. It might also be opportune to consider the need to characterise populations who might benefit from the targeted interventions because of functional metabolic polymorphisms; this, of course, is already done for some inborn errors of metabolism. Consideration of these aspects of functionality highlights the relative lack of knowledge about childhood nutrition. For example, what would be the physiological basis of using functional effects to monitor nutrition in infants fed breast milk or breast milk substitute, or to resolve issues related to the subsequent diversification of the diet (weaning) (Kramer \& Kakuma, 2002)? The increasing appreciation of the cognitive benefits of exogenous docosahexaenoic acid in formula-fed infants exemplifies the use of visual acuity as a functional effect, but further insight is demonstrating functional effects at cellular levels that might, in due course, provide tighter markers with which to monitor docosahexaenoic acid needs and the adequacy of supply (Uauy et al. 2001).

Differential effects according to the timing and duration of inadequate I supply on psychomotor and cognitive function are well appreciated. There is now concern that analogous issues might arise from $\mathrm{Fe}$ deprivation. Compared with a 20-year-old, a neonate has $10 \%$ and a 10-year-old has $50 \%$ of the ultimate amount of brain Fe. Young rat models show irredeemable psychomotor defects arising from Fe deficiency at weaning. There is concern that this might also apply to human infants. If so, is there a critical period at which Fe supply must be adequate, and what is the relevant action threshold for Fe deficiency and functional marker for this (Grantham-McGregor \& Ani, 2001)? There are similar important challenges relating to the timing and duration of exposure to potential allergens, lipids and cholesterol.

The fundamental problem with tackling these issues is the availability of appropriate functional effects or markers. The functional outcomes and their markers in the determination of ideal food intakes are most useful if they are closely related temporally and mechanistically to the supply of the nutrient or food component.

There is a need for markers to enable studies to be based on more immediate outcomes and to be derived from appropriate tissues. The markers need not be closely aligned to the target function if there is a clear mechanistic pathway linking the two. Thus there is arguably a role for genetics, genomics, proteomics and metabolomics in assessing functional effects as well as detecting early warning of potential adverse effects in the context of nutritional safety (Ordovas, 2002; German et al. 2003). A better understanding of homeostatic mechanisms and their mediators would provide markers of an adequate range of intakes within which one might reasonably expect nutrients to be achieving their optimal effects. The current investment in nutritional genomics, or, more particularly, post-genomic molecular function, should be expected to provide markers of functional effects more relevant and specific to nutrients and food components of interest.

The development of new markers for functional effects in children would enable a new generation of hypothesisdriven intervention studies that would address both the functional and homeostatic outcomes. Within this concept, I think there would be the opportunity to explore early nutrition and adult disease and generate data upon which to base recommendations for nutrient requirements, and safety.

\section{Acknowledgements}

This work was supported by a grant from the Nutritional Needs of Children Task Force of the European branch of the International Life Sciences Institute (ILSI Europe). Industry members of this task force are Barilla, Coca-Cola, Danone Vitapole, Friesland Coberco, Masterfoods, Nestlé and Numico. Further information about ILSI Europe can be obtained from $+32(0) 27710014$ or 
info@ilsieurope.be. The opinions expressed herein are those of the authors and do not necessarily represent the views of ILSI and ILSI Europe.

\section{References}

Barker DJ (1994) Maternal and fetal origins of coronary heart disease. J R Coll Physicians Lond 28, 544-551.

Bellisle F (2004) Effects of diet on behaviour and cognition in children. Br J Nutr 92, Suppl. 2, S227-S232.

Committee for Medical Aspects of Food Policy (1991) Dietary Reference Values of Food Energy and Nutrients for the United Kingdom. Department of Health Report on Health and Social Subjects no. 41. London: The Stationery Office.

Deckelbaum RJ \& Williams CL (2001) Childhood obesity: the health issue. Obes Res 9, Suppl. 4, 239S-243S.

Diplock AT, Aggett PJ, Ashwell M, Bornet F, Fern EB \& Roberfroid MB (1999) Scientific concepts of functional foods in Europe: consensus document. Br J Nutr 81, Suppl. 1, S1-S27.

Expert Group on Vitamins and Minerals (2003) Safe Upper Levels for Vitamins and Minerals. London: The Food Standards Agency.

Geleijnse JM, Grobbee DE \& Hofman A (1990) Sodium and potassium intake and blood pressure change in childhood. $\mathrm{Br}$ Med J 300, 899-902.

Geleijnse JM, Hofman A, Witteman JCM, Hazebroek AAJM,
Valkenburg HA \& Grobbee DE (1996) Long-term effects of neonatal sodium restriction on blood pressure. Hypertension 29, 913-917.

Gerber M (2001) The comprehensive approach to diet: a critical review. J Nutr 131, 3051S-3055S.

German JB, Roberts M-A \& Watkins SM (2003) Genomics and metabolomics as markers for the interaction of diet and health: lessons from lipids. J Nutr 133, 2078S-2083S.

Godfrey KM \& Barker DJ (2001) Fetal programming and adult health. Public Health Nutr 4, 611-624.

Grantham-McGregor S \& Ani C (2001) A review of studies on the effect of iron deficiency on cognitive development in children. J Nutr 131, 649S-666S.

Kramer MS \& Kakuma R (2002) The Optimal Duration of Exclusive Breastfeeding; A Systematic Review. Geneva: WHO.

Koletzko B, Aggett PJ, Bindels JG, Bung P, Ferre P, Gil A, Lentze M, Roberfroid M \& Strobel S (1998) Growth, development and differentiation: a functional food science approach. Br J Nutr 80, Suppl. 1, S5-S45.

Ordovas JM (2002) Gene-diet interaction and plasma lipid responses to dietary intervention. Biochem Soc Trans 30, $68-73$.

Uauy R, Hoffman DR, Peirano P, Birch DG \& Birch EE (2001) Essential fatty acids in visual and brain development. Lipids 36, 885-895. 